\title{
A point-of-care test increases same-day referral and reduces DKA in children with new-onset type 1 diabetes
}

Farrah Rodrigues,

Michele A O'Connell, Mary White

\begin{abstract}
Background and objective
Same-day referral for tertiary management of suspected childhood type 1 diabetes (T1D) is recommended as best practice. The aim of this study was to review recent primary care practice in managing suspected paediatric T1D and its impact on clinical outcomes.
\end{abstract}

\section{Methods}

This was a retrospective cross-sectional study of referral patterns and clinical outcomes of youth aged $<18$ years with new-onset T1D at two tertiary metropolitan paediatric diabetes centres.

\section{Results}

Almost half (73 out of 155) of children and adolescents later diagnosed with T1D had delayed referral; $56 \%$ (41 out of 73 ) had additional pre-referral investigations. Point-of-care (POC) blood/urine glucose testing was associated with increased same-day referral (odds ratio [OR] 14.6; 95\% confidence interval $[\mathrm{CI}]: 5.9,36.3$, $P<0.001)$, reduced pre-referral investigations (OR $0.4 ; 95 \% \mathrm{Cl}: 0.2$, $0.9, P=0.02)$ and reduced diabetic ketoacidosis (DKA) rates $(60.3 \%$, compared with $27.8 \%, P<0.001)$.

\section{Discussion}

POC testing facilitated early tertiary referral and reduced DKA rates and unnecessary investigations in children and adolescents with new presentation of T1D.
TYPE 1 DIABETES (T1D) is the most common form of diabetes in the paediatric age group and its incidence is increasing. ${ }^{1,2}$ The incidence of diabetic ketoacidosis (DKA), a potentially avoidable and preventable complication of $\mathrm{T} 1 \mathrm{D}$, is also on the rise, with $15-67 \%$ of new T1D presenting in DKA. ${ }^{3}$ DKA is associated with significant morbidity, increased healthcare expenditures and a mortality risk. ${ }^{4}$ In addition to acute complications, DKA has a long-term impact on cognitive function, including IQ compromise and persistent loss of short-term memory. ${ }^{5}$

The combination of osmotic symptoms and/or symptoms and signs of ketosis (Table 1), together with a random point-of-care (POC) blood glucose level ( $\geq 11.1 \mathrm{mmol} / \mathrm{L})$ or glycosuria, are sufficient to make the clinical diagnosis of T1D and no further investigations are required for the majority. ${ }^{1,6}$ A confirmatory laboratory glucose is required where suspicion is raised on the basis of POC glucose measurement; this may be undertaken by the specialist/ emergency department (ED) team before commencing insulin and should not delay referral. Nevertheless, high rates of delayed referral and thus DKA have been reported internationally. ${ }^{7}$

In an Australian context, Craig et al reported that $79 \%$ of new-onset T1D paediatric patients had previously presented to their general practitioner (GP) within one week of diagnosis, without immediate referral for specialist management. ${ }^{3}$ Of those youth who were seen by a GP with symptoms suggestive of T1D (Table 1), 31\% had pre-referral laboratory investigations; DKA rates were higher in this cohort. ${ }^{3}$ The International Society of Paediatric and Adolescent Diabetes (ISPAD) clinical practice consensus guidelines recommend same-day referral for paediatrician/ paediatric endocrinologist management of suspected T1D. ${ }^{1}$ This has been highlighted and addressed in Australian primary care management guidelines ${ }^{8}$ as previous studies have shown that same-day referral significantly reduced the rates of DKA. ${ }^{3,4,9,10}$

Although adverse outcomes of delayed presentation have been well documented, clinical experience suggested that a large proportion of newly diagnosed youth with T1D still experience a delay in referral for paediatrician/paediatric endocrinologist management.

The aim of the study was to establish the frequency of same-day referral and presentation for paediatrician/paediatric endocrinologist management, to explore factors associated with same-day referral to ED or for additional investigation and to assess the potential impact of these practices on clinical outcomes. It was hypothesised that a large proportion of youth in the Melbourne metropolitan area with new-onset T1D experienced delay in establishing the diagnosis, that pre-referral investigations are frequently requested and that same-day referral 
would decrease rates and severity of DKA at presentation. The premise of this study was to seek to identify any aspect of the early management of T1D that is amenable to promotion within the primary care setting to improve patient outcomes, and to consider options for a collaborative approach to best practice care of these young people.

\section{Methods}

A retrospective cross-sectional study was conducted at the two tertiary paediatric centres in Melbourne, Australia - the Royal Children's Hospital ( $\mathrm{RCH})$ and Monash Children's Hospital (MCH) which together manage approximately $80 \%$ of paediatric cases of T1D in Victoria. ${ }^{11}$ Ethical approval was received at both participating sites (RCH HREC LCL-17/01 and MH HREC 16303Q) before study commencement. Data (demographic, medical and pathology) were collected and analysed for the period July 2015 to July 2016 with data collection occurring from July 2016 to May 2017. Participants were eligible for inclusion if they were aged 1-18 years and newly diagnosed with T1D at one of the two tertiary centres from 1 July 2015 to 30 June 2016. New presentations of non-T1D forms of diabetes and those new to the studied centres but with established T1D were excluded (Figure 1).

Data were extracted from GP referral letters, medical notes and presenting hospital laboratory records and put into Microsoft Excel. Face validity applies to the data collection with an attempt to confirm accuracy through triangulation of data sources. ${ }^{12}$ Same-day referral was defined as referral to a secondary/tertiary paediatric centre for ongoing management on the same day as a GP review where the diagnosis was suspected. Patients who were referred by a GP on the same day as their review but were registered as seen the next day (eg because of ED wait times) were included as same-day

\section{Table 1. Common presentations of type 1 diabetes}

\begin{tabular}{|c|c|c|}
\hline Symptoms & & Commonly interpreted as \\
\hline \multirow{6}{*}{$\begin{array}{l}\text { Osmotic } \\
\text { symptoms - } \\
\text { secondary to } \\
\text { hyperglycaemia }\end{array}$} & Polyuria & Urinary tract infection \\
\hline & Polydipsia & Hot weather \\
\hline & Nocturia & $\begin{array}{l}\text { Drinking too much before bed, urinary } \\
\text { tract infection }\end{array}$ \\
\hline & Secondary enuresis & $\begin{array}{l}\text { Drinking too much before bed, urinary } \\
\text { tract infection }\end{array}$ \\
\hline & Tiredness/lethargy & Iron deficiency/school related \\
\hline & Change in behaviour/mood & Age related/puberty, infection \\
\hline \multirow{4}{*}{$\begin{array}{l}\text { Ketotic symptoms } \\
\text { resulting in } \\
\text { acidosis }\end{array}$} & Nausea and abdominal pain & Constipation \\
\hline & Vomiting & Gastroenteritis \\
\hline & Change in appetite & Growth related \\
\hline & Weight loss & Growth spurt, reduced appetite \\
\hline
\end{tabular}

\section{Signs}

Dehydration - dry mouth, tongue, tachycardia

\begin{tabular}{ll}
\hline Ketotic breath & Sore throat, gum disease \\
\hline Hyperventilation in the context of ketosis & Respiratory tract infection \\
\hline Kussmaul breathing - as ketosis worsens & Respiratory tract infection \\
\hline Altered conscious state & Infection \\
\hline
\end{tabular}

referral. ISPAD criteria for DKA, namely hyperglycaemia (random plasma glucose $>11 \mathrm{mmol} / \mathrm{L}$ ), acidosis (venous $\mathrm{pH}<7.3$ or serum bicarbonate $<15 \mathrm{mmol} / \mathrm{L}$ ) and ketonemia or ketonuria were applied. ${ }^{13}$ Severity of presentation was documented as per ISPAD guidelines (Table 2). ${ }^{13}$

For each participant the following data were recorded: confirmed diagnosis of T1D, age at diagnosis, sex, documented history of GP review with symptoms suggestive of T1D (yes/no), same-day referral versus delayed referral, interval in days between review and presentation to an ED, POC blood/urine glucose testing with GP (yes/no), pre-referral investigation (yes/no); DKA at presentation (yes/no); observed complications of DKA (Glasgow Coma Score $<15$, cerebral oedema, intensive care unit admission), and length of hospital stay (days).

Data were coded and analysed using STATA 13.0. Continuous and categorical outcomes were analysed using Student's t-test and chi-squared analyses respectively. Variables that may have influenced the decision and referral pathway were analysed using logistic regression analyses. A $P$ value of $<0.05$ was considered significant.

\section{Results}

Two hundred and nine possible cases were identified. Three cases who were new to the studied centres but who had established T1D were excluded. Data were collected on 206 subjects (93 out of 206 male, 45\%; Figure 1).

The mean (standard deviation) age at presentation was 9.5 (4.5) and 9.5 (4.0) years in patients referred on the same day and those with delayed referral respectively. One hundred and fifty-five patients ( 155 out of $206,75 \%$ ) were seen by a GP before ED presentation; delayed referral was documented in 73 out of 155 individuals (Figure 1). The median interval between the time of first GP review and referral for ED presentation was two days (1-31 days). Overall, 27\% (20 out of 73) of referrals were delayed by more than five days and $10 \%$ (7 out of 73 ) were seen by their GP multiple times before the diagnosis was made. 


\section{9 new referrals to $\mathrm{RCH} / \mathrm{MCH}$ during study period}

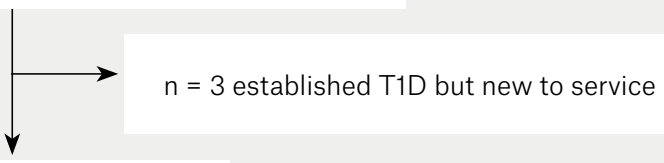

206 included in data analysis
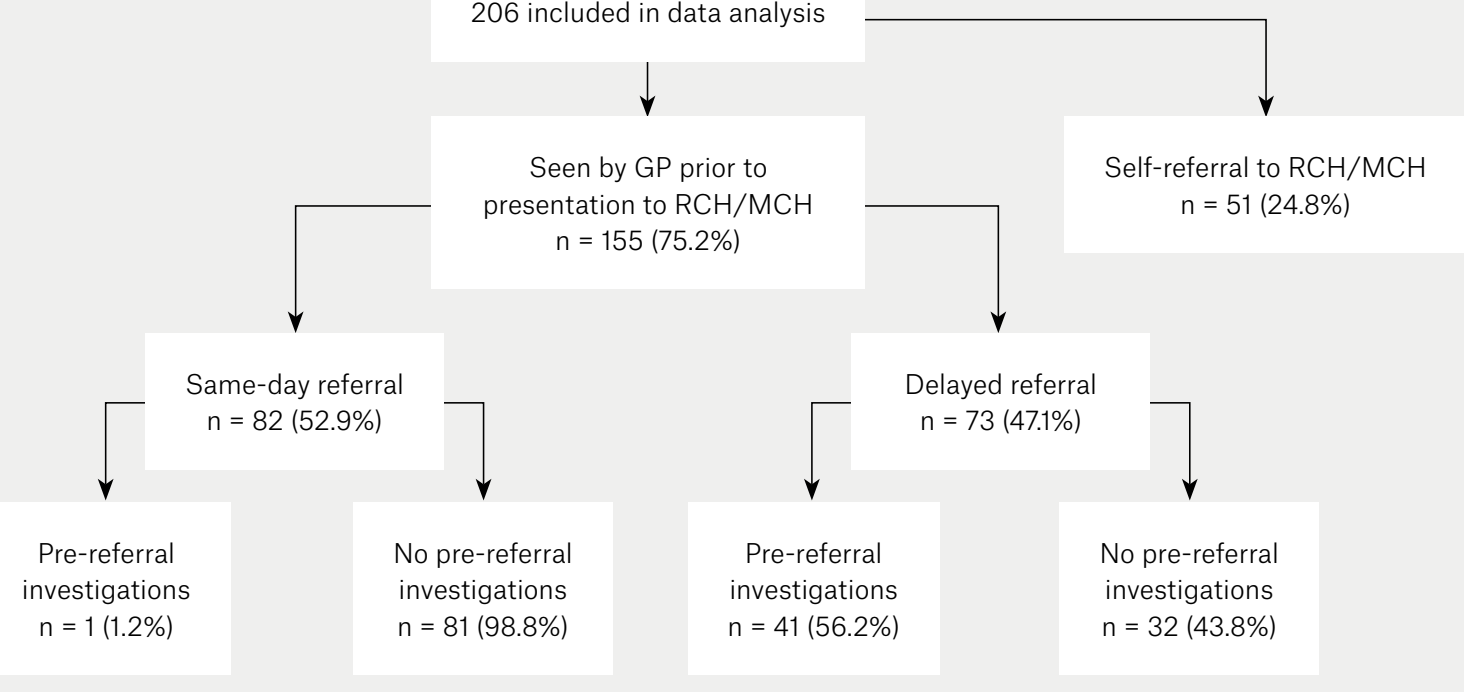

Figure 1. Referral pathway of patients included in this retrospective study

GP, general practitioner; $M C H$, Monash Children's Hospital; RCH, Royal Children's Hospital; T1D, type 1 diabetes

Pre-referral investigations were significantly more common in those who had delayed referral (41 out of 73 , compared with 1 out of 82 in those with same-day referral to ED) (56.2\%, compared with $1.2 \%, P<0.001$; refer to Figure 1 and Table 3). Of the cohort with delayed referral and pre-referral investigation, $90 \%$ (37 out of 41 ) had a fasting glucose level, oral glucose tolerance test or both. Youth with delayed referral without pre-referral investigation were reported by parents/carers to have been given an alternative initial diagnosis including gastroenteritis, constipation, urinary tract infection, oral candida, viral illness and failure to thrive.

The characteristics of youth who presented in DKA are shown in Table 2. DKA was noted in $\mathrm{n}=82$ out of 206 (40\%), of whom $52 \%$ ( $n=43$ out of
82) had moderate to severe acidosis. Of these 43 individuals, 15 (18.2\%) required intensive care admission and management. The rates $(45 \%$, compared with $54 \%, P=0.1$ ) and severity (moderate 25\%, compared with 33\%, $P=0.2$; severe $17.9 \%$ compared with $18.2 \%, P=0.6$ ) of DKA were similar between the same-day referral and delayed referral groups (Table 2). In patients with moderate to severe DKA, the median interval between the time of first GP review and referral for ED presentation was three days (1-30 days).

POC blood/urine glucose testing was independently associated with a higher likelihood of same-day referral and lower pre-referral investigation (Table 4). Those with primary care POC testing had significantly lower rates of DKA $(27.8 \%$, compared with $60.3 \%)$ than those without POC testing $(P<0.001)$.

\section{Discussion}

This study showed that, of children and adolescents with newly diagnosed T1D in the Melbourne metropolitan area, just over half $(52 \%)$ of those who were reviewed by a GP had the recommended same-day referral for paediatrician/ paediatric endocrinologist management. Importantly, an easily accessible measure, namely POC blood/urine glucose testing, was associated with a significant increase in the odds of same-day referral and reduced the odds of the patient developing moderate to severe DKA.

A significant majority (75\%) of the overall cohort saw a GP before presentation to a tertiary centre. However, these data highlight ongoing high frequency of DKA at the point of initial GP presentation, delayed referral and arguably unnecessary pre-referral investigation in Australian youth presenting with likely T1D. 
In the early stages of T1D development, the signs and symptoms (Table 1 ) are often subtle and non-specific. ${ }^{14}$ Distinguishing children with T1D from the large number with minor undifferentiated illness is therefore difficult, ${ }^{14}$ particularly since GPs see a vast variety of presentations with short consultation times for clinical assessment and decision making. Patient factors may also contribute, as parents may predominantly describe symptoms of ketosis rather than osmotic symptoms; the alternative diagnoses reported in the cohort in the present study are conditions more frequently seen in paediatric primary care presentation than T1D and are similar to those reported elsewhere. ${ }^{7}$ Usher-Smith et al described that all children with T1D present with one or more symptoms of polyuria, polydipsia, tiredness and weight loss (Table 1), and suggest that clinicians should remain alert to the 4Ts of T1D (toilet, thirsty, tired and thinner).${ }^{14}$ Targeted questioning for these $4 \mathrm{~T}$ symptoms in children and adolescents presenting with symptoms such as abdominal pain, nausea or vomiting, which may be manifestations of ketosis, is therefore recommended.

In contrast to an ED setting, paediatric T1D and DKA are infrequent presentations to a given GP or practice, unlike type 2 diabetes (T2D), which represents $85 \%$ of all diabetes presenting in adulthood ${ }^{15}$ and is frequently managed in the primary care setting alone. The distinction between the referral pathways is that the urgency for emergency management for individuals with adult-onset $\mathrm{T} 2 \mathrm{D}$ is less pressing and typically the laboratory investigations that comprised the majority of pre-referral investigation in this cohort are required to establish the diagnosis. Familiarity with this diagnostic work-up may have influenced the decision-making pathway in this paediatric cohort. In contrast to adults, T1D predominates in children and adolescents and an elevated blood/ urine glucose level is sufficient to prompt referral to the ED of a specialist paediatric diabetes centre. ${ }^{1}$ Crucially, because of the insulinopaenia and potential for early development of DKA, the diagnosis of $\mathrm{T} 1 \mathrm{D}$ should be assumed in all paediatric and adolescent patients and they should be referred on the same day as their GP review. Similarly, for these reasons, referral for outpatient clinic review is not appropriate in the case of suspected T1D. While family factors outside of a primary clinician's control may separately influence decisions or practicalities around timing of presentation, reinforcement by GPs of the potentially serious health-related sequelae of delayed presentation may help to facilitate early presentation to ED. Confirmatory diagnostic laboratory testing can then be performed in the specialist ED.

It can be hypothesised that those who presented with osmotic symptoms but who appeared clinically well at their initial GP review provoked some uncertainty around the clinical diagnosis, prompting pre-referral investigation. Of this group,

\section{Table 2. Severity and clinical features of diabetic ketoacidosis}

\begin{tabular}{|c|c|c|c|c|c|}
\hline & & Overall n (\%) & \multicolumn{3}{|c|}{ Seen by general practitioner $\mathbf{n}(\%)$} \\
\hline & & \multirow[t]{3}{*}{$82 / 206(40)$} & & \multicolumn{2}{|c|}{$61 / 155$ (39) } \\
\hline & & & SDR & DR & $P$ value \\
\hline & & & $n=28$ & $n=33$ & \\
\hline \multirow{3}{*}{$\begin{array}{l}\text { Diabetic } \\
\text { ketoacidosis } \\
\text { severity* }\end{array}$} & Mild & $39(47.6)$ & $16(57.1)$ & $16(48.5)$ & 0.7 \\
\hline & Moderate & 25 (30.5) & $7(25)$ & $11(33.3)$ & 0.2 \\
\hline & Severe & $18(21.9)$ & $5(17.9)$ & $6(18.2)$ & 0.6 \\
\hline \multicolumn{2}{|c|}{ Glasgow Coma Score $<15$} & $16(19.5)$ & $3(3.9)$ & $6(8.1)$ & 0.2 \\
\hline \multicolumn{2}{|c|}{ Intensive care unit admission } & $15(18.2)$ & $4(4.9)$ & $2(2.7)$ & 0.5 \\
\hline \multicolumn{2}{|c|}{ Cerebral oedema } & $1(1.2)$ & $0(0)$ & $1(1.4)$ & 0.3 \\
\hline
\end{tabular}

*Diabetes ketoacidosis severity as defined by the International Society of Paediatric and Adolescent Diabetes guidelines: mild $-p H<7.3$ or bicarbonate $<15 \mathrm{mmol} / \mathrm{L}$; moderate $-p H 7.2$ or bicarbonate

$<10 \mathrm{mmol} / \mathrm{L}$; severe $-\mathrm{pH}<7.1$ or bicarbonate $<5 \mathrm{mmol} / \mathrm{L}$

$D R$, delayed referral; $S D R$, same-day referral

Table 3. Subgroup characteristics of patients with general practitioner review before specialist presentation

\begin{tabular}{lrrrr}
\hline & Overall & SDR & DR & P value \\
\hline Age in years, median (SD)* & $155(75)$ & $82(52.9)$ & $73(47)$ & \\
\hline Length of stay in days, mean (SD)* $^{*}$ & 9.5 & $9.5(4.5)$ & $9.5(4.0)$ & 0.9 \\
\hline Male, $\mathrm{n}(\%)^{\dagger}$ & 3.65 & $3.6(1.6)$ & $3.7(1.7)$ & 0.5 \\
\hline POC blood glucose test, $\mathrm{n}(\%)$ & $69(44)$ & $43(52)$ & $26(36)$ & 0.06 \\
\hline POC urine glucose test, $\mathrm{n}(\%)$ & $92(59)$ & $68(82.9)$ & $24(32.9)$ & $<0.001$ \\
\hline POC blood/urine glucose test, $\mathrm{n}(\%)$ & $14(9)$ & $7(8.5)$ & $7(9.6)$ & 0.8 \\
\hline Pre-referral investigation, $\mathrm{n}(\%)^{\dagger}$ & $102(65)$ & $73(89)$ & $29(39.7)$ & $<0.001$ \\
\hline${\text { Diabetic ketoacidosis, } \mathrm{n}(\%)^{\dagger}}^{\dagger}$ & $42(27)$ & $1(1.2)$ & $41(56.2)$ & $<0.001$ \\
\hline
\end{tabular}

*Group differences tested with Student's t-test (continuous) analysis

${ }^{\dagger}$ Group differences tested using chi-squared (categorical) analysis

$D R$, delayed referral; $P O C$, point of care; $S D$, standard deviation; SDR, same-day referral 
Table 4. Logistic regression analyses of the factors associated with referral decision making

\begin{tabular}{|c|c|c|c|c|c|c|}
\hline & \multicolumn{3}{|c|}{$\begin{array}{l}\text { Same-day referral } \\
\qquad n=82(52.9 \%)\end{array}$} & \multicolumn{3}{|c|}{$\begin{array}{l}\text { Pre-referral investigations } \\
\qquad n=41(47 \%)\end{array}$} \\
\hline & Odds ratio & $95 \% \mathrm{CI}$ & $P$ value & Odds ratio & $95 \% \mathrm{CI}$ & $P$ value \\
\hline $\begin{array}{l}\text { Point-of-care glucose/ } \\
\text { urinalysis }\end{array}$ & 14.6 & $5.9,36.3$ & $<0.001$ & 0.4 & $0.2,0.9$ & 0.02 \\
\hline $\begin{array}{l}\text { Moderate to severe } \\
\text { diabetic ketoacidosis }\end{array}$ & 1.5 & $0.6,3.9$ & 0.4 & 0.4 & $0.2,0.10$ & 0.05 \\
\hline Age at presentation & 0.9 & $0.9,1.1$ & 0.5 & 1.1 & $0.95,1.1$ & 0.4 \\
\hline \multicolumn{7}{|c|}{$\begin{array}{l}\text { Note: Data were analysed using logistic (odds ratio, } 95 \% \mathrm{Cl} \text { and } \mathrm{P} \text { value) regression for categorical } \\
\text { independent variables. } \\
\mathrm{Cl} \text {, confidence interval }\end{array}$} \\
\hline
\end{tabular}

the vast majority $(90 \%)$ had a fasting glucose, oral glucose tolerance test or both performed, suggesting that the diagnosis of diabetes was correctly considered. Same-day referral without the need for confirmatory laboratory investigations (which were associated with delayed presentation in almost all cases) is the preferred course of action in this scenario. Despite numerous studies, the progression of T1D from onset of symptoms to the development of DKA is difficult to predict for a given individual. ${ }^{16}$ Therefore, the above-mentioned subgroup is arguably the most likely to gain benefit from direct referral to an ED without additional investigation, as the time taken to arrange and review investigations may have affected the subsequent development of ketosis and acidosis. Concerningly, over $50 \%(n=17$ out of 33$)$ of participants who had seen a GP but had delayed referral of up to three days presented to ED in either moderate or severe DKA with $18 \%$ ( $n=6$ out of 33 ) of participants showing a reduced conscious state, and one with clinically significant cerebral oedema (Table 2). The present data indicate that a change in practice to routinely perform POC testing to confirm suspicion of T1D and facilitate same-day referral may improve clinical outcomes in children with T1D. The researchers acknowledge that in younger children the practicalities of obtaining a urine sample for analysis may pose a challenge for timely diagnosis; fingerpick blood glucose level testing is equally accessible to these age groups.

It is concerning that DKA rates at first presentation of T1D are so high, at almost $40 \%$. Of note in those who were referred to ED on the same day as their GP review, 43\% had moderate to severe DKA, indicating that a significant proportion were already very unwell at the time of presentation to their GP. This is a public health awareness issue and beyond the scope of this report. The relatively high rate of first presentations to a GP with symptoms of established DKA has likely influenced the finding that, unlike previous studies, ${ }^{3}$ same-day referral was not associated with lower rates of DKA than those with delayed referral in this cohort.

Moving forward, the dilemma is how to address this issue in a collaborative, acceptable, sustainable and cost-effective manner. Untargeted community education campaigns do not appear to effect change. ${ }^{17}$ Despite the published data in Australian medical journals ${ }^{3,18}$ and integration of this information into GP training programs, the difficulty remains that the take-home message is attenuated by the relative rarity of paediatric T1D in the primary care setting. Local clinical practice guidelines and clinical referral pathways have attempted to address experience gaps in this area, but the uptake and effect of these initiatives is unknown. ${ }^{19}$
Inclusion of paediatric common presentations in The Royal Australian College of General Practitioners' Choosing Wisely recommendations is an example of a sustainable, wide-reaching and cost-effective mechanism by which crucial information can be disseminated but understandably, high- rather than low-yield presentations have been included to date. Alternatively, integration of a clinical decision support system within practice computer programs may be useful as an automatic prompt to highlight the need for same-day referral for suspected new-onset T1D. The feasibility of this technology is currently being explored by the authors.

\section{Limitations}

The limitations of this study include the relatively small sample size with data collected retrospectively over only a one-year period, which could affect external validity and statistical conclusions. However, this cohort represents approximately $80 \%$ of new presentations with paediatric T1D in Victoria. ${ }^{11}$

Additional limitations include cases from a metropolitan setting only, although arguably same-day referral and ED presentation is more easily facilitated in this environment. Patient/ family factors that may have affected timing of presentation could not be ascertained and would be best addressed on a prospective basis. The suspected diagnosis of T1D at referral was obtained from the referral letter where available. However, if a referral letter was not available, documentation of GP referral or parental report of same was considered as evidence of referral source, although it is not possible to definitively confirm the accuracy of this information. Retrospective data extraction also meant family report of previous GP review and outcomes could not be corroborated, which may be subject to recall bias.

\section{Conclusion}

Same-day referral for commencement of insulin is best practice when managing 
new-onset paediatric T1D. This study showed that POC blood/urine glucose testing in primary care is associated with significantly higher rates of same-day referral, fewer (arguably unnecessary) pre-referral investigations and, most importantly, lower DKA rates at diagnosis of T1D in children and adolescents. Early use of these inexpensive and readily available tools can significantly affect important clinical outcomes for these young patients. The use of clinical decision support systems for the diagnosis of rare and uncommon conditions may be a sustainable mechanism by which this important information can be widely disseminated.

\section{Implications for general practice}

- Although much less commonly encountered in general practice than $\mathrm{T} 2 \mathrm{D}, \mathrm{T} 1 \mathrm{D}$ is the most common cause of diabetes in children and adolescents. As T1D is characterised by insulin deficiency with the potential for rapid progression to DKA, same-day referral for paediatrician/paediatric endocrinologist management is recommended.

- T1D can masquerade as other conditions; youths do not always present with classical osmotic symptoms and when symptoms of ketosis have developed, osmotic symptoms may not feature in their presenting complaint. Targeted questioning is recommended.

- In the context of symptoms suggestive of T1D, POC tests indicating either glycosuria (+/- ketonuria) or blood glucose level $\geq 11.1 \mathrm{mmol} / \mathrm{L}$ are sufficient to confirm likely T1D and obviate the need for any additional investigations before referral to a tertiary centre.

- POC testing was associated with significantly fewer additional pre-referral investigations, increased likelihood of same-day referral and lower rates of DKA.

- To optimise outcomes, universal POC testing is recommended to facilitate diagnosis and early referral where new-onset paediatric T1D is considered in primary care.

\section{Authors}

Farrah Rodrigues MBBS, DCH, BSc, PGDipSci, Paediatric Endocrinology Fellow, Department of Endocrinology and Diabetes, Monash Children's Hospital, Vic

Michele A O'Connell MB BAO BCh, MRCPI, FRACP, $M D$, Paediatric Endocrinologist, Department of Endocrinology and Diabetes, The Royal Children's Hospital, Vic; Honorary Research Fellow, Murdoch Children's Research Institute, Vic; Honorary Senior Fellow, The University of Melbourne, Vic Mary White MB BAO BCh, MRCPI, FRACP, $M D$, Paediatric Endocrinologist, Department of Endocrinology and Diabetes, The Royal Children's Hospital, Vic; Research Officer, Murdoch Children's Research Institute, Vic; Honorary Senior Fellow, The University of Melbourne, Vic

Competing interests: None.

Funding: None.

Provenance and peer review: Not commissioned, externally peer reviewed.

\section{Correspondence to:}

farrahrodrigues@gmail.com

\section{References}

1. Mayer-Davis EJ, Kahkoska AR, Jefferies C, et al. ISPAD clinical practice consensus guidelines 2018: Definition, epidemiology, and classification of diabetes in children and adolescents. Pediatr Diabetes 2018:19 Suppl 27:7-19. doi: 10.1111/ pedi.12773.

2. Lawrence SE, Cummings EA, Gaboury I, Daneman D. Population-based study of incidence and risk factors for cerebral edema in pediatric diabetic ketoacidosis. J Pediatr 2005;146(5):688-92. doi: 10.1016/j.jpeds.2004.12.041.

3. Craig ME, Wong $\mathrm{CH}$, Alexander J, Maguire AM, Silink M. Delayed referral of new-onset type 1 diabetes increases the risk of diabetic ketoacidosis. Med J Aust 2009;190(4):219. doi: 10.5694/j.1326-5377.2009.tb02356.x.

4. Jefferies CA, Nakhla M, Derraik JG, Gunn AJ, Daneman D, Cutfield WS. Preventing diabetic ketoacidosis. Pediatr Clin North Am 2015;62(4):857-71. doi: 10.1016/j.pcl.2015.04.002.

5. Cameron FJ, Scratch SE, Nadebaum C, et al. Neurological consequences of diabetic ketoacidosis at initial presentation of type 1 diabetes in a prospective cohort study of children. Diabetes Care 2014;37(6):1554-62. doi: 10.2337/dc13-1904.

6. Chiang JL, Maahs DM, Garvey KC, et al. Type 1 diabetes in children and adolescents: A position statement by the American Diabetes Association. Diabetes Care 2018;41(9):2026-44. doi: 10.2337/ dci18-0023.

7. Bui H, To T, Stein R, Fung K, Daneman D. Is diabetic ketoacidosis at disease onset a result of missed diagnosis? J Pediatr 2010;156(3):472-77. doi: 10.1016/j.jpeds.2009.10.001.

8. The Royal Australian College of General Practitioners and Australian Diabetes Association. Emergency management of hyperglycaemia in primary care. East Melbourne, Vic: RACGP, 2018.

9. Usher-Smith JA, Thompson MJ, Sharp SJ, Walter FM. Factors associated with the presence of diabetic ketoacidosis at diagnosis of diabetes in children and young adults: A systematic review. BMJ 2011;343:d4092. doi: 10.1136/bmj.d4092.

10. King BR, Howard NJ, Verge CF, et al. A diabetes awareness campaign prevents diabetic ketoacidosis in children at their initial presentation with type 1 diabetes. Pediatr Diabetes 2012;13(8):647-51. doi: 10.1111/j.13995448.2012.00896.x
11. de Bock M, Jones TW, Fairchild J, Mouat F, Jefferies $C$. Children and adolescents with type 1 diabetes in Australasia: An online survey of mode of care, workforce and outcomes. J Paediatr Child Health 2019;55(1):82-6. doi: 10.1111/jpc.14122.

12. Dixon N, Pearce M. Guide to ensuring data quality in clinical audits. London, UK: HQIP, 2011.

13. Wolfsdorf JI, Allgrove J, Craig ME, et al. ISPAD clinical practice consensus guidelines 2014 Diabetic ketoacidosis and hyperglycemic hyperosmolar state. Pediatr Diabetes 2014;15 Suppl 20:154-79. doi: 10.1111/pedi.12165.

14. Usher-Smith JA, Thompson MJ, Zhu H, Sharp SJ, Walter FM. The pathway to diagnosis of type 1 diabetes in children: A questionnaire study. BMJ Open 2015;5(3):e006470. doi: 10.1136/bmjopen2014-006470.

15. Australian Bureau of Statistics. National health survey: First results, 2014-15. Belconnen, ACT: ABS, 2015.

16. Couper JJ, Haller MJ, Greenbaum CJ, et al. ISPAD clinical practice consensus guidelines 2018: Stages of type 1 diabetes in children and adolescents. Pediatr Diabetes 2018;19 Suppl 27:20-27. doi: 10.1111/pedi.12734.

17. Derraik JGB, Cutfield WS, Maessen SE, et al. A brief campaign to prevent diabetic ketoacidosis in children newly diagnosed with type 1 diabetes mellitus: The NO-DKA study. Pediatr Diabetes 2018;19(7):1257-62. doi: 10.1111/pedi.12722.

18. Sabin MA, Cameron FJ, Werther GA. Type 1 diabetes: Still the commonest form of diabetes in children. Aust Fam Physician 2009;38(9):695-97.

19. HealthPathway. Health Pathways Melbourne. Parkville, Vic: NWMPHN, 2020. Available at https://nwmphn.org.au/health-systems-capacitybuilding/healthpathways [Accessed 20 July 2020]. 